\section{HUMAN GENETICS}

\section{Polymorphic Man}

Professor Harry Harris delivered the ninth Darwin lecture last week at the British Museum (Natural History), and his choice of subject was a field in which he is justly famous, the population genetics of enzymes and proteins.

The realization that men do not all share the same stock of proteins came, twenty years ago, with Pauling's demonstration that sickle cell anaemics possess an aberrant form of haemoglobin in their red blood cells. Since then, of course, many protein polymorphisms have been detected in man and other species. More than a hundred variant forms of haemoglobin alone are known, most of them differing by a single amino-acid substitution from the normal molecule, and presumably arising by a single base change in its gene.

Professor Harris described the work he and his group have done in assessing the extent of protein variation in human populations. Of nineteen arbitrarily chosen enzymes, chiefly from red cells, six were shown by starch gel electrophoresis to possess frequent genetic variants. Variant forms of peptidase A appeared among Negroes but not in Europeans, while the variants of adenylate kinase appeared among Europeans but not Negroes.

These results allow a tentative calculation of the number of protein polymorphisms in a human individual. About one third of all amino-acid substitutions provide a change in electric charge, and it is probably only these substitutions that are detected by starch gel electrophoresis. The enzymes studied so far have a heterozygosity of five per cent per locus. Multiplying this by three to allow for undetected variants, and taking fifty thousand as a modest estimate of the number of genetic loci in man, the conclusion is that each individual may be heterozygous in about eight thousand loci. Clearly each person is unique in his enzyme constitution.

It is unlikely that many of these polymorphisms arose spontaneously, but it is extremely difficult to elucidate the selection pressures that may have led to their origin. Professor Harris said that the only protein variant understood in terms of selective advantage remains the sickle-cell haemoglobin of Centra] Africa. Sickle-cell heterozygotes are less prone to malaria than normal individuals, though homozygotes suffer, and indeed rarely survive to reproductive age.

The difficulty in interpreting selection pressures in human populations is, of course, compounded by the speed of social change through which we are living. The incidence and age distribution of mortality are changing profoundly, so that the selective agencies which shaped the pattern of today's polymorphisms may well have lost their selective significance.

\section{PLANT GROWTH}

\section{Hormones in Order}

\section{from a Correspondent}

A MAJOR advance in understanding of the chemical control of plant growth was the realization that plant growth hormones do not operate alone, but interact in various complex ways. This makes the control of plant growth easier to visualize, but does nothing to simplify the experimental elucidation of its mode of action. In a recent issue of Nature (221, 1267; 1969), Eastwood, Taverner and Laidman described experimental evidence for the cooperation of gibberellic acid and kinetin (a synthetic cytokinin, 6-furfurylaminopurine) in the germination of wheat seeds. Gibberellic acid influences many activities in the plant such as flowering and seed germination, while the cytokinins are involved in cell division and expansion.

Although it is not known in detail how any plant growth hormone works, regulation of the mechanism by which enzymes are made in the cell must form part of the answer. Strict control of enzyme mediated growth could thus be maintained by the combined action of several hormones. Wheat or barley seeds consist of a starchy food-store, the endosperm separated from the embryo by a layer of cells containing granules of protein, the aleurone layer. In the early stages of germination, insoluble starch in the endosperm is broken down into soluble sugars which can be assimilated by the embryo. It has been known for some time that the aleurone layer can be induced to synthesize the enzyme $\alpha$-amylase, responsible for the breakdown of starch to sugars in the endosperm, by gibberellic acid. In natural conditions, gibberellic acid will come from the embryo. If the embryo is removed, enzyme synthesis can still be induced in the aleurone layer by adding gibberellic acid to the remains of the seed, but only if the seeds have been soaked in water for one to three days. It is clearly possible that other hormones may have become active in the seed tissue during this time. Dr Laidman has noted that an extract of the starchy endosperm will stimulate metabolic activity in the aleurone layer, and that natural cytokinins can be used in place of this endosperm extract. As long ago as 1898, H. T. Brown and F. Escombe (Proc. Roy. Soc., 63, 3) wrote: "During normal germination the endosperm is not wholly passive, but takes some share with the embryo in preparing the reserve materials for the use of the young plant". Dr Laidman's experiment seems to have verified this earlier assertion. Sections of aleurone layer were cut from seeds that had been soaked for only four hours. These were incubated in growth solutions to which gibberellic acid, kinetin or nothing had been added. Large amounts of $a$-amylase were secreted into the solution in all three cases, but there was no difference in amount between the samples treated with hormone and the controls. The same aleurone sections were then transferred to fresh media, containing either gibberellic acid or no additional substance. The sections incubated initially in kinetin and then in gibberellic acid showed twice as much $\alpha$-amylase activity when compared with the other treatments.

These results suggest that there is a sequential cooperation of growth hormones in the induction of enzyme activity in germinating wheat. 'The implication is that cytokinin from the endosperm stimulates physiological activity in the aleurone layer, possibly by organizing the genetic machinery for enzyme production. Synthesis of a-amylase is then initiated by gibberellic acid produced by the embryo and transported into the aleurone layer.

Further developments in this work should prove interesting, but, looking afresh at the results of Brown and Escombe after 70 years, it seems that there are few new ideas in plant physiology, only elegant verification of the old. 